\title{
Considerations of Ethics While Allowing Flexibility for Trainees: The Model and The Rationale for the Model of the Yale Global Mental Health Program
}

\author{
Carla Marienfeld ${ }^{1}$ (D)
}

Received: 7 August 2015 / Accepted: 10 September 2015 / Published online: 30 September 2015

(C) Academic Psychiatry 2015

To the Editor:

The purposes of the Yale Global Mental Health Program (YGMHP) are to ensure that the residents are knowledgeable about Global Mental Health (GMH) and to provide additional elective options for those with a special interest in GMH. Trainees often come expecting flexibility in the types of experiences they will have so as to best fit their career trajectory. Our program advocates for the primary role of the resident in seeking out experiences and collaborations, with support and mentorship from our faculty, as a way to promote more long-term, career-spanning relationships with the partner site. This can be done at sites that have longstanding relationships with the residency, department, or medical school. It can also lead to new relationships that, with support and mentorship for the resident to develop, nurture, and maintain these collaborations, are essential for a career in GMH.

The program explicitly seeks long-term collaborative relationships for the residents that go beyond time-limited provision of clinical care for chronic mental illnesses in the LAMIC. An advantage of having a fixed site with an ongoing relationship is to facilitate safe and consistent experiences for residents. However, the limitations include a potential lack of initiative on the part of the resident to explore and identify areas of interest for their future career, to seek out new mentors and colleagues, and to establish their own partnerships and mutually beneficial projects or to continue work started prior to residency. Allowing residents to become the primary coordinator of his/her experience also increases and allows for a gain in knowledge of the culture, language, and system of care in the partner site.

Carla Marienfeld

marienfeld@gmail.com; carla.marienfeld@yale.edu

1 Yale University School of Medicine, New Haven, CT, USA
The YGMHP program seeks to meet the training needs for GMH work conducted during residency and beyond, including education about general topics in GMH, awareness about the host site, mentorship for the project they wish to complete (research, teaching, policy development, etc.), and training in ethical issues. A key feature is the provision of mentorship for the work that they will be doing both at home and abroad. Residents benefit from an oversight committee comprising faculty and other residents that provides additional support for the program. Without the passion and creativity from this group, developing partnerships and funding from professional organizations, academic institutions, NGO's, grants, and foreign governments for our residents' experiences would have lagged.

The impact of the YGMHP is evident by the increase in the number of core curriculum topics on GMH, funded resident international experiences, resident scholarly works, and residents reporting YGMHP as a factor in their decision to rank Yale residency [1]. Despite these successes, challenges remain. While we have some consistent funding, identification of long-term sustained funding for both resident salary/benefits and for international travel and projects remains a key factor. Ethical considerations about using international experiences during residency and medical school to visit foreign lands rather than to promote health or building healthcare and research capacity are minimized in this model. However, we have ethical obligations to help ensure the resident abroad is properly mentored and supervised such that they are not in situations beyond their training or expertise, and this can be challenging to secure with each new site.

\section{Reference}

1. Marienfeld C, Rohrbaugh RM. Impact of a global mental health program on a residency training program. Acad Psychiatry. 2013;37(4):276-80. 